\title{
The Influence of Visual Distraction on Awareness Test
}

\author{
Rachel Yen-Ting Lo ${ }^{1,2}$, Mein-Woei Suen 1,3,4 \\ ${ }^{1}$ School of Psychology, Institute of Clinical Psychology, Chung Shan Medical University, Taichung City, Chinese \\ Taipei \\ ${ }^{2}$ Department of Psychology, Simon Fraser University, Burnaby, Canada \\ ${ }^{3}$ Chung Shan Medical University Hospital, Taichung City, Chinese Taipei \\ ${ }^{4}$ Social and Gender Issue Research Center, Chung Shan Medical University Hospital, Taichung City, Chinese \\ Taipei \\ Email: blake5477@yahoo.com.tw
}

Received 6 July 2014; revised 8 August 2014; accepted 20 August 2014

Copyright (C) 2014 by authors and Scientific Research Publishing Inc.

This work is licensed under the Creative Commons Attribution International License (CC BY). http://creativecommons.org/licenses/by/4.0/

\section{(c) (i) Open Access}

\begin{abstract}
In everyday situation, there are so many events happening around our lives. Although human beings are capable of perceiving many events happening at the same time, it is not possible for individuals to pay attention to every little detail that they face in this complex environment. In this study, the effect of visual distraction on awareness test was evaluated. The hypothesis was that the presence of visual distraction via laser lights will lower the score on awareness test, whereas a higher score on awareness test would occur when laser lights were absent. The study evaluated 3 male and 14 female university undergraduate students' performance on awareness test and predicted that their performance would be affected by distractions. In the end, data analysis obtained a supportive result to the hypothesis. The study brought up more significant issues between distraction and awareness which also drew more interests for researchers in different fields.
\end{abstract}

\section{Keywords}

Distraction, Awareness, Perception

\section{Introduction}

The topic of attention has evoked psychologists' interests for many years already, and it is becoming a more popular issue in different fields. Many researchers have explored this topic in various ways. Storm and Hernandez (2007) viewed attention from a cognitive perspective. They were particularly interested in eye movements because "eye movements determine what individual can see"; whether the object in front of an individual is a ball, a pencil, or anything else, they all require attention in order to be seen. That is, eye movements provide 
controlled access to objects in the visual world. Correspondingly, eye movements are also capable to remove access to objects in the visual world (Storm \& Hernandez, 2007).

Lambert and Hockey (1986) also viewed the attention in a similar way. Yet, they introduced the term "selective attention" to be the main focus of their research, in which, they believed that human beings have the ability to detect stimuli from different attributes or dimensions at the same time. Therefore individuals have to find their ways to focus on what they want to pay attention to and not get distracted by other surrounding stimuli.

If human are designed to perceive various stimuli at the same time, what if "too many" stimuli appear at once? Would we really able to catch them all? In Macdonald and Lavie (2008)'s research, the "loading" of the stimulus became another important issue. According to the perceptual load theory, distractor can affect reaction time and or neural activity. In other words, when high distractor is present, the perception would be desensitized, and vice versa (Macdonald \& Lavie, 2008). When one requires taking in multiple stimuli at one time, his or her information process capacity would relatively slow down. Sometimes the intake of multiple stimuli could block object that's especially important at the specific moment and context, an irreversible harm could occur as a result. One way to avoid desensitizing of perception is to limit the person's demand on eye processing, as well as, lower the loading of perceptual information intake.

Cheal and Chastain (2002) also conducted an experiment particularly on visual selective attention. They suggested that the efficiency of selective attention was associated with the types of the targets; meaning, there was a greater chance to get distracted if the target and the distractive source (stimuli) were the same type. In Cheal and Chastain (2002)'s study, they used the color red and red to be the same type of stimuli. Yantis and Hillstrom (1994) also indicated that attention was captured in visual search by the appearance of a new perceptual object even when the object was equivalent to the object that one was originally looking at. This is the main reason that I design my experiment with a combination of video and laser lights rather than video and sounds, because they are visually the same type!

Attention is also a hot topic for educators. Doyle, Anderson, \& Halcomb (1976) demonstrated a study on attention deficits and visual distraction. The results showed that learning disabled children tend to be more easily distracted, hyperactive, or impulsive in many different tasks than normal children. They were more likely to have a lower correct detection rate and higher false alarm or random response than normal children as well (Doyle, Anderson, \& Halcomb, 1976). Therefore learning disability children are always the ones who get special care and most attention at school; most schools nowadays have special education for these children.

Parents worry about distractive stimuli in the complex and dangerous traffic environment where can possibly take away their children's lives. However, Tabibi and Pfeffer (2007) indicated that the ability to distinguish safe and dangerous situations in traffic environment and ignoring distracting stimuli can be trained and improved with increasing age. Therefore it is very important for the educators and parents to deliver the important lessons about the correct road sense when the children are still young. Nevertheless, we cannot only rely on the children to learn the significance of road sense. The safety of the road is the responsibility of each citizen. Many countries have established policy to restrain drivers texting and talking with the phone in the car, in order to reduce distractions that could possibly cause fatal harm during driving.

After reviewing these valuable literatures, one would conclude visual distraction would affect one's attention and awareness. An experiment based on the concepts mentioned above was conducted. Not only so, several improvements and precious interventions for education and road safety were also suggested for further study.

\section{Method}

\subsection{Participants}

The subjects of the study were the students who enrolled in Psychology 201 course at Simon Fraser University in the semester of spring 2010. The experiment was carried out in a tutorial class which consisted with 17 students ( 3 males and 14 females). Of the 17 students, there were 8 Caucasian, 5 Asian, 3 Indian, and 1 Black; with an average age of 20 years old. The attendance was strongly encouraged to avoid any mark deduction. Yet, their participation was absolute voluntary, anonymous, and confidential. Students were allowed to withdraw any time if they desire to do so.

\subsection{Materials}

It was an independent group design. Participants were randomly assigned into two groups and each participant 
was required to participate in only one of two conditions. Data was collected from participants' questionnaire from both groups were compared and analyzed to see if the results support the hypothesis.

The independent variable was the distractive stimuli, which was the laser light in this study. It was manipulated in both control and experimental group conditions. In the control group (Group A), there was no presence of laser lights. The participants were asked to watch a video and then fill out the questionnaire. In the experimental group (Group B), the laser lights were presented by swinging around the wall against the screen during the video was playing. With the presence of distraction of laser lights, it was predicted that the laser lights could seize individuals' attention that they might scatter their attention to the laser lights for a few seconds even if they tried hard to focus on the video.

The dependent variable was the scores that each participant got from the questionnaire. The questionnaire was consisted 7 items, in multiple choice questions, asking about small details of the video. The video was chosen to play was called "21 Random Thoughts" by Dan Brown. The content of the video was neither about psychology nor anything to do with topics such as attention or distraction. However, this study was meant to test individuals' awareness. Therefore in order to answer these questions correctly, participants had to pay fully attention when watching the video. The dependent variable was measured in a total out of seven. It indicated the performance level of each participant; the higher the scores they got, the more focused they were. The video and questionnaire had been verified by expertise examination, whose reliability and validity were sufficient to be used in this study.

\subsection{Procedure}

As the beginning of the experiment, a brief instruction about the study was giving out to the participants. And then a consent form was passed around the class for the participants to sign, on the agreements of willing to participant in this research. Next, each participant was randomly assigned to either Group A or B. In order to avoid the two groups affecting each other, only one group could go at a time and the other had to wait quietly outside till the other group finished.

Group A was asked to do the experiment first. Each participant got a sheet of questionnaire facing down along with a piece of scratch paper. Since this was meant to test participants' awareness not memorization, they were allowed to use the scratch paper to assist them draw down whatever they saw in the video. In order to make the independent variable more on visual distraction orientated, Experimenter intended to turn the volume off during the entire video was playing. As soon as they finished watching the video, they could start answering the questionnaire. The copies of questionnaire were then collected to be analyzed for this study. Now Group A had completed their task. They were told to go outside and let Group B came in to the experiment. After Group B had seated, each of them got the same questionnaire and scratch paper that Group A had. The video was played in mute as well; this made the whole study more consistent. Nevertheless, the independent variable was manipulated in Group B. Experimenter used a laser pen to swing around the wall against the screen, trying to distract their attention while they watching the video. The laser pen was handled carefully and used it in proper manner; made sure it was not hurting anybody's eyes.

And of course, the ethics checklist was signed by both my professor and Teacher Assistant and attached in the appendices package so the participants do not need to worry about the laser lights would cause any harm to them. Once they finished watching the video, they were asked to fill out the questionnaire as another part of data to be analyzed and compared with Group A.

After the experimenter had collected all sets of papers from each subject, the experiment was ended with a verbal debriefing that the purpose and the hypothesis of the study were stated. All subjects also got a hard copy of debrief form with the experimenter's phone number and email where they could reach if they have concerns about the study.

Both of the control and experimental groups were conducted in same day, same tutorial class, within the same time frame. But most importantly, the two groups had to be done one at a time. In the consent form, there were not statements about the true purpose of the study as well as the indication of the laser lights. These were the ways to avoid biases. Moreover, the participants were randomly assigned to one of the two groups. Therefore the entire experiment was completely randomized.

It was predicted that the presence of laser light distractions would lower the performance score on the awareness test because it was too hard for the participants of Group B to pay full attention to the video when there were other stimuli also appearing at the same time. Therefore the scores of Group B would be lower than Group A. 


\section{Hypothesis}

It was predicted that the presence of laser light distractions would lower the performance score on the awareness test because it was too hard for the participants of Group B to pay full attention to the video when there were other stimuli also appearing at the same time. Therefore the scores of Group B would be lower than Group A.

\section{Result}

The data was collected in one single tutorial class with a total of 17 subjects. All 17 subjects completed the questionnaire, and no one had dropped out. The 17 subjects had been randomly assigned into two groups, Group A had 8 subjects and Group B had 9 subjects). However, their questionnaires were collected all together to compare and analyze. Thus, participants' performance on awareness test was measured based on the 7-multiple choice questionnaire. The higher the scores they had, the better the performance level they did on the awareness test.

Individuals' score was calculated by summing up the score that they achieved on each question. Then, the total score of each group (control and experimental group) was calculated by adding up all individual scores of the particular group. For Group A the total score was 46, whereas, for Group B was 43. The difference of the total scores between the two groups was 3. A mean score was the sum of the scored and divided the number of subjects. Group A's mean $(M=5.75)$ was higher than Group B's mean $(M=4.78)$. The difference of the means between the two groups was 0.97. Standard deviation was also calculated for the two groups. Group A's standard deviation $(S D=1.75)$ was also higher than Group B's standard deviation $(S D=1.56)$. The difference of the standard deviation between the two groups was 0.19. I noticed that the differences of these scores between the Group A and Group B were not that distinctive. Yet, when examining the percentages of the number of people got six or more questions correct, the differences were clearly to be seen. For Group A, there were 5 out of 8 people (62.5\%) scored at least 6 questions correct; while Group B only had 3 out of 9 people (33\%) scored at least 6 questions correct.

The statistics suggested that all three scores (the total score, the mean, and the standard deviation) were higher in Group A, as well as the percentages of the number of people scored six or more questions correct were also higher in Group A. Even though the differences of each score between the two groups were not significantly large, the results seemed to be in favor of the hypothesis.

\section{Discussion}

The main purpose of the study was to investigate the effect of visual distraction via laser lights on an awareness test. The independent variable was the presence of laser lights and the dependent variable was the scores that the participants got for the awareness test questionnaire. The hypothesis was that the presence of laser lights would lower the scores on the questionnaire because the laser lights could possibly scatter one's attention when there were too many stimuli occurring at the same time. The design of the experiment was based on Venkatesan and Haaland (1968)'s study on divided attention. In their experiment, the subjects were shown a videotape containing a television commercial. They were visually distracted during the exposure. After the videotape was played, they were asked to recall the brand name and product type of the commercial.

In this experiment, one tutorial class was divided into two groups and collected data of those two groups. Expectedly, the results had successfully supported the hypothesis that the control group had achieved all three numbers (the total score, the mean, and the standard deviation) on awareness questionnaire higher than the experimental group.

Although the hypothesis had proved by the data analysis in the end, there were some strengths and weaknesses in the experiment needed a further review. For the strengths, the study was supported by the literature, and had gone through a pilot study. It was randomized; the flow of the experiment was appropriate and quite smoothly, and the choice of laser lights as visual distraction was unique and well-functioned in the experiment. The length of questionnaire was relatively short and relevant to the video they were watching, so the participants did not need to know extra knowledge for completing the questions. Moreover, the confounding variables were minimized by the control procedures throughout the entire experiment.

However, the weaknesses were more concerned. First of all, the scratch paper that had been handed out in the beginning seemed unnecessary that there were only a few people used it as reference when doing the question- 
naire. Because of the short video, it did not require a lot of memory for the participants to remember. The pace of the video was quite fast; the participants hardly had time to draw down what they saw in the meanwhile as well. Secondly, as mentioned above, the choice of video playing in this study was not that good because of its fast pace. Even if the participants watched it under normal condition, without any kinds of distraction, they would probably miss some small details about the video. Therefore decision on choice of video should be made more careful for future study. Instead of using a fast pace video, a moderate pace video would be more applicable. This could also minimize some confounding variables that might affect the results. The negative about multiple choice questions was that participants could simply take a guess even if they do not know the answer. It was unknown for the experimenter if any participants really did take a guess when answering the question, but if somebody really did, the results would not be as accurate.

In addition, muting the sound also made the independent variable more sufficiently focused on visual distraction (Hillstrom \& Chai, 2006); even if the participants missed seeing some parts of the video, they could still know the contents by hearing when the video was played with sounds. Furthermore, in order for everyone to see the video clearly a projector was used.

Even though the result of the study was quite predictable, the mechanism behind based on the literature review had inspired the author about the direction of future research. Author was also encouraged to seek out substantial interventions on improving school environment and primarily assisting children with special need with the help of attention-enhancing skills. This will not only improve the learning outcome of the children in the same classroom as a whole, it will also protect the rights of the children who do not need as much help. On the other hand, the road safety issue can drastically threaten each person's life. With the knowledge obtained about attention and distraction, academia could perhaps incorporate with public services such as Government (more particularly, Ministry of Transportation, etc.) or local library or community center. As a role of researcher, we can possibly offer workshops to deliver basic knowledge of road sense to the public (for example, what the sources of distractor are and how they would affect us on the road).

\section{Acknowledgements}

We thank Shu-Jun Huang for exploring ideas with us and Amy Liao for editing this experiment write-up.

\section{References}

Cheal, M., \& Chastain, G. (2002). Efficiency of Visual Selective Attention Is Related to the Type of Target. Psychological Research/Psychologische Forschung, 66, 110-115. http://dx.doi.org/10.1007/s00426-001-0083-0

Doyle, R., Anderson, R., \& Halcomb, C. (1976). Attention Deficits and the Effects of Visual Distraction. Journal of Learning Disabilities, 9, 48-54. http://dx.doi.org/10.1177/002221947600900109

Hillstrom, A. P., \& Chai, Y. (2006). Factors That Guide Or Disrupt Attentive Visual Processing. Computers in Human Behavior, 22, 648-656. http://dx.doi.org/10.1016/j.chb.2005.12.003

Lambert, A., \& Hockey, R. (1986). Selective Attention and Performance with a Multidimensional Visual Display. Journal of Experimental Psychology: Human Perception and Performance, 12, 484-495. http://dx.doi.org/10.1037/0096-1523.12.4.484

Macdonald, J. P., \& Lavie, N. (2008). Load Induced Blindness. Journal of Experimental Psychology: Human Perception and Performance, 34, 1078-1091. http://dx.doi.org/10.1037/0096-1523.34.5.1078

Storm, B., \& Hernandez, A. (2007). Cognitive Consequences of Asymmetrical Visual Distraction. Journal of General Psychology, 134, 415-434. http://dx.doi.org/10.3200/GENP.134.4.415-434

Tabibi, Z., \& Pfeffer, K. (2007). Finding a Safe Place to Cross the Road: The Effect of Distractors and the Role of Attention in Children's Identification of Safe and Dangerous Road-Crossing Sites. Infant and Child Development, 16, $193-206$. http://dx.doi.org/10.1002/icd.509

Venkatesan, M., \& Haaland, G. (1968). Divided Attention and Television Commercials: An Experimental Study. Journal of Marketing Research, 5, 203-205. http://dx.doi.org/10.2307/3150029

Yantis, S., \& Hillstrom, A. (1994). Stimulus-Driven Attentional Capture: Evidence from Equiluminant Visual Objects. Journal of Experimental Psychology: Human Perception and Performance, 20, 95-107.

http://dx.doi.org/10.1037/0096-1523.20.1.95 
Scientific Research Publishing (SCIRP) is one of the largest Open Access journal publishers. It is currently publishing more than 200 open access, online, peer-reviewed journals covering a wide range of academic disciplines. SCIRP serves the worldwide academic communities and contributes to the progress and application of science with its publication.

Other selected journals from SCIRP are listed as below. Submit your manuscript to us via either submit@scirp.org or Online Submission Portal.
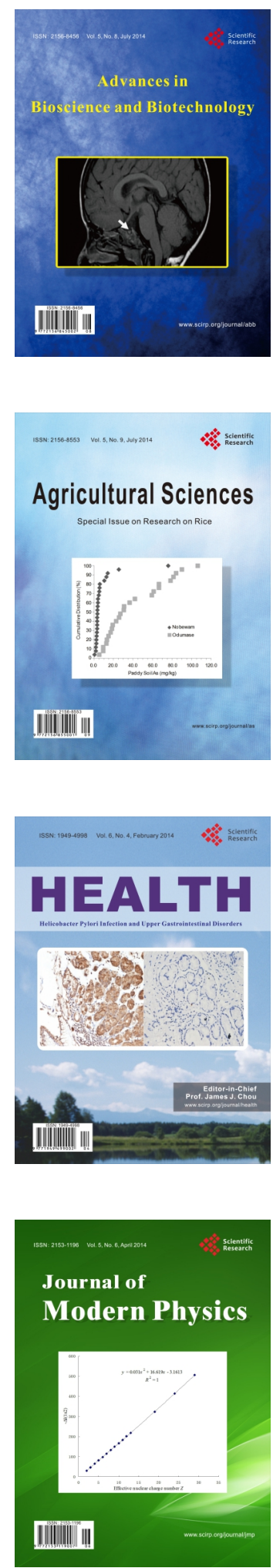
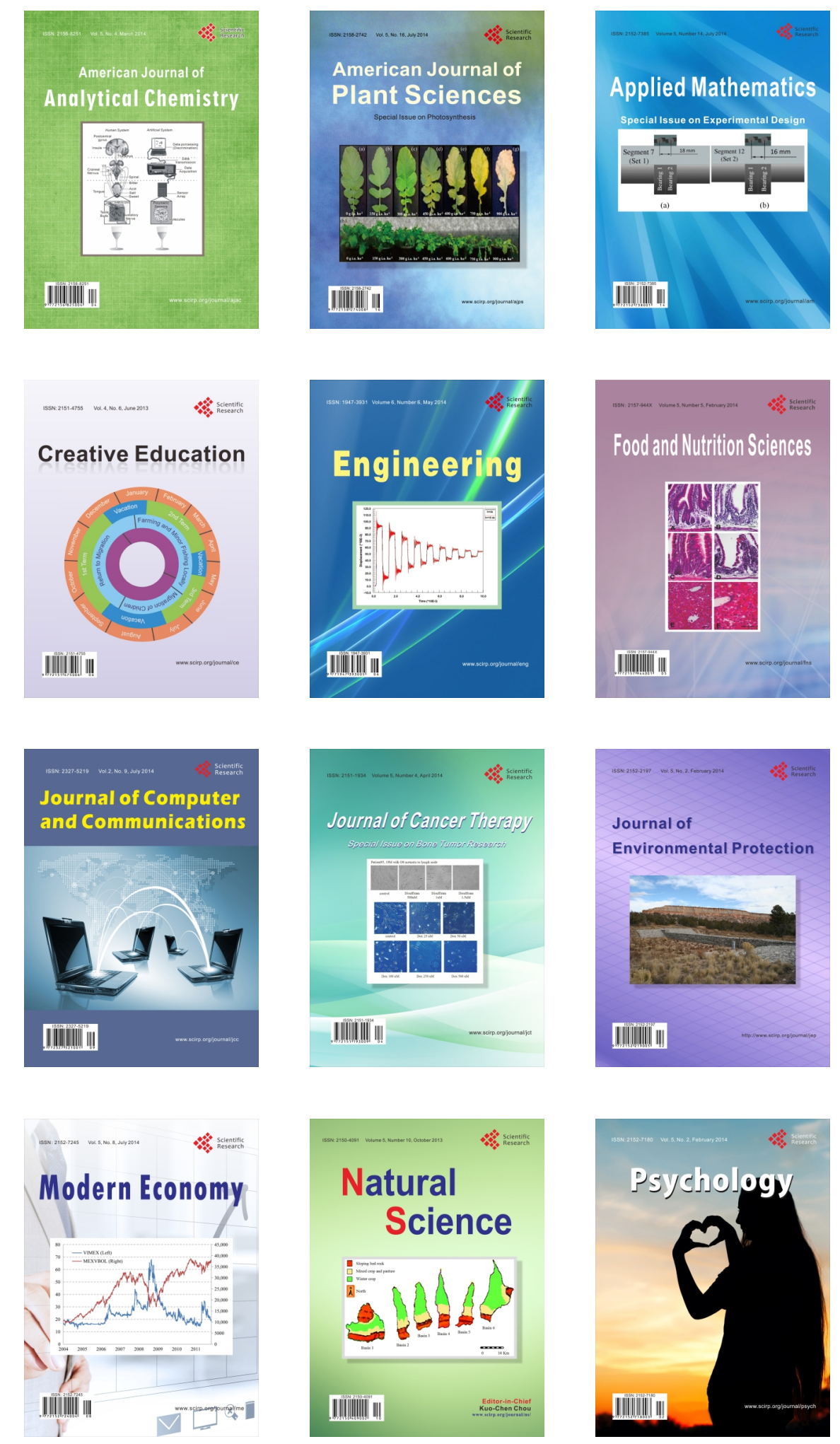